\title{
Geometric Information and Rational Parametrization of Nonsingular Cubic Blending Surfaces
}

\author{
Minghao Guo, Tieru Wu, and Shugong Zhang
}

Key Laboratory of Symbolic Computation and Knowledge Engineering (Ministry of Education), School of Mathematics, Jilin University, Changchun 130012, China

Correspondence should be addressed to Minghao Guo, guomh08@mails.jlu.edu.cn

Received 21 March 2011; Accepted 7 June 2011

Academic Editor: Ke Chen

Copyright (C) 2011 Minghao Guo et al. This is an open access article distributed under the Creative Commons Attribution License, which permits unrestricted use, distribution, and reproduction in any medium, provided the original work is properly cited.

The techniques for parametrizing nonsingular cubic surfaces have shown to be of great interest in recent years. This paper is devoted to the rational parametrization of nonsingular cubic blending surfaces. We claim that these nonsingular cubic blending surfaces can be parametrized using the symbolic computation due to their excellent geometric properties. Especially for the specific forms of these surfaces, we conclude that they must be $F_{3}, F_{4}$, or $F_{5}$ surfaces, and a criterion is given for deciding their surface types. Besides, using the algorithm proposed by Berry and Patterson in 2001, we obtain the uniform rational parametric representation of these specific forms. It should be emphasized that our results in this paper are invariant under any nonsingular real projective transform. Two explicit examples are presented at the end of this paper.

\section{Introduction}

In the areas of computer graphics and geometric modeling, we may sometimes encounter the problems about cubic surfaces. A real nonsingular cubic surface $V(f)$ can be defined either by the implicit form, that is, the real zeros of a polynomial equation $f\left(x_{1}, x_{2}, x_{3}\right)=0$ of degree 3 in $R^{3}$, or by the rational parametric form, that is,

$$
x_{1}=\frac{f_{1}\left(y_{1}, y_{2}, y_{3}\right)}{f_{4}\left(y_{1}, y_{2}, y_{3}\right)}, \quad x_{2}=\frac{f_{2}\left(y_{1}, y_{2}, y_{3}\right)}{f_{4}\left(y_{1}, y_{2}, y_{3}\right)}, \quad x_{3}=\frac{f_{3}\left(y_{1}, y_{2}, y_{3}\right)}{f_{4}\left(y_{1}, y_{2}, y_{3}\right)},
$$

where $f_{1}, \ldots, f_{4}$ are homogeneous polynomials and $y_{1}, y_{2}$, and $y_{3}$ are parameters. Both forms are appropriate for solving different types of problems. For example, in the geometric 
modeling systems, the implicit form offers many advantages when performing geometric operations like union, intersection, blending, and warping. Besides, the rational parametric form is preferred in the free-form surface modeling, because it guarantees manifoldness and provides a way of generating points within the surface. So, the automatic transition between these two forms is very important.

Generating a rational parametric representation of an algebraic surface (called parametrization for short) is always difficult, even impossible sometimes, while the reverse process called implicitization is always solvable (such as using Gröbner basis method). Most of the parametrization algorithms for nonsingular cubic surfaces are based on geometric information of nonsingular cubic surfaces, such as the existence of 27 lines on nonsingular cubic surfaces [1]. In 1987, Sederberg and Snively parametrized cubic surfaces in terms of biquadratic polynomials using pairs of skew lines on the surfaces in [2]. This method is further developed by Bajaj et al. in [3] and Polo-Blanco and Top in [4]. In 2001, Berry and Patterson unified the implicitization and parametrization of a nonsingular cubic surface using Hilbert-Burch theorem in [5]. In 2007, Chen et al. presented an alternative method for parametrizing quadric and cubic surfaces based on the theory of $\mu$-basis in [6].

This paper is devoted to nonsingular cubic blending surfaces in [7-9], which meet two quadratic surfaces with $G^{1}$ continuity. The blending surfaces have attracted more and more attention in recent years and have been used in particular for filling surface holes, smoothing corners and edges, and making computer animation. Wu and Cheng in [10] discussed the parametrization of the special blending surfaces defined by

$$
f=\left(b_{1}\left(x_{2}-d_{2}\right)+b_{2}\left(x_{1}-d_{1}\right)\right)\left(x_{1}^{2}+x_{2}^{2}+x_{3}^{2}-r^{2}\right)-\left(x_{1}-d_{1}\right)\left(x_{2}-d_{2}\right),
$$

where $b_{1}, b_{2}, d_{1}, d_{2}$, and $r$ are real numbers. We further develop their results in a more general way in this paper. We consider successively two classes of these nonsingular cubic blending surfaces: the specific forms and the general forms.

(i) For the specific forms, we conclude that they must be $F_{3}, F_{4}$, or $F_{5}$ surfaces, and present a criterion to decide their surface types. Besides, using the algorithm proposed in [5], we obtain their uniform rational parametric representation.

(ii) For the general forms, although they do not have the analogous concise properties as the specific forms do, we can still come to the conclusion that their rational parametrizations can be computed using the symbolic computation.

Additionally, it should be pointed out that our results in this paper are invariant under any nonsingular real projective transform.

The rest of the paper is organized as follows. Section 2 recalls some geometric information of nonsingular cubic surfaces and introduces the constructions for nonsingular cubic blending surfaces. Section 3 is devoted to the specific forms of nonsingular cubic blending surfaces, and we study in detail the geometric information on them and their uniform rational parametric representation. Section 4 discusses the analogous geometric properties of the general forms of nonsingular cubic blending surfaces as the specific forms. Two explicit examples are presented in Section 5. Finally, we conclude the paper in Section 6. 
Table 1: Some geometric information of nonsingular cubic surfaces (derived from [14]).

\begin{tabular}{lccccc}
\hline Surface type & $F_{1}$ & $F_{2}$ & $F_{3}$ & $F_{4}$ & $F_{5}$ \\
\hline Number of real lines & 27 & 15 & 7 & 3 & 3 \\
Number of real tritangent planes & 45 & 15 & 5 & 7 & 13 \\
Number of real components & 1 & 1 & 1 & 1 & 2 \\
\hline
\end{tabular}

\section{Notations and Preliminaries}

For the convenience of applications, we always consider the problem in $\mathrm{R}\left[x_{1}, x_{2}, x_{3}\right]$. Let $V(f)$ denote the algebraic surface determined by the polynomial equation $f\left(x_{1}, x_{2}, x_{3}\right)=0$. Assume that $F$ is a set of polynomials, and denote by $V(F)$ the set of solutions of the system of all polynomials in $F$. Let $\langle g, h\rangle$ be the ideal generated by the polynomials $g$ and $h$.

\subsection{Geometric Information of Nonsingular Cubic Surfaces}

In 1849, Cayley and Salmon published the famous theorem that there are 27 lines lying completely on a nonsingular cubic surface. Every line on a nonsingular cubic surface is met by ten others. A plane containing three of the lines is called a tritangent plane. There are 45 such planes on a nonsingular cubic surface. Nonsingular cubic surfaces can be divided into 5 species $F_{1}, F_{2}, \ldots, F_{5}$ with respect to the number of real lines and real components. See Table 1 for more details.

Remark 2.1. Most of the parametrization algorithms lose effectiveness for $F_{5}$ surfaces, since the $F_{5}$ surfaces have no real one-to-one parametrization. Thus, we will not address nonsingular cubic blending surfaces of type $F_{5}$ in this paper.

The computation of lines on a nonsingular cubic surface is our starting point for the analysis and parametrization of the surface, since we could know other geometric information of the surface from Table 1 once the number of real lines on the surface is verified.

Sederberg showed how to compute lines on a nonsingular cubic surface in [11]. Assume that we have a nonsingular cubic surface given by its implicit equation $f\left(x_{1}, x_{2}, x_{3}\right)=$ 0 . The parametrization of a line with unknown coefficients is $l(t):=\left(t, x_{20}+x_{21} t, x_{30}+x_{31} t\right)$. Substituting the parametric equation of the line into the implicit equation of the surface yields an equation $f(l(t))$ of degree 3 in the parameter $t$. If this equation is identically zero, that is, all the coefficients vanish simultaneously, it guarantees that the line lies entirely on the surface. In this way, the problem of finding a line on a nonsingular cubic surface is transformed into the problem of solving a system of four nonlinear equations in four unknowns. We can find at least one solution of this system using Gröbner basis method or some other techniques.

If one line $l(t)$ is known, we can use this line to find other lines on the surface. We take a pencil of planes through $l(t)$ and intersect it with the surface. The intersection consists of $l(t)$ and a residual conic $\mathcal{C}$. The condition for $\mathcal{C}$ to degenerate into a pair of lines is that the determinant of its Hessian vanishes. The determinant of the Hessian of $\mathcal{C}$ is a polynomial of degree 5 . Each of the 5 roots corresponds to a plane in which the residual intersection is degenerate. After computing the roots of this polynomial, it is possible to get other lines on the surface. 
Remark 2.2. It is relatively expensive to compute one line on a nonsingular cubic surface. Besides, since quintic equations can only be solved numerically, not analytically, we could not obtain exact lines on the surface. However, nonsingular cubic blending surfaces in this paper just avoid these obstacles.

\subsection{Nonsingular Cubic Blending Surfaces}

In this subsection, we briefly introduce the constructions of nonsingular cubic blending surfaces. Suppose that we are given two quadratic surfaces $V\left(g_{1}\right), V\left(g_{2}\right)$, and two associated clipping planes $V\left(h_{1}\right), V\left(h_{2}\right)$. Without loss of generality, we assume that $V\left(h_{1}\right)$ and $V\left(h_{2}\right)$ intersect. Denote $h_{3}=x_{i}$, where $i \in\{1,2,3\}$ such that $V\left(h_{1}, h_{2}, h_{3}\right)$ is an isolated point in $\mathrm{R}^{3}$.

$\mathrm{Wu}$ et al. in [12] concluded that if the coefficients of $g_{1}, g_{2}, h_{1}$, and $h_{2}$ satisfy some certain conditions, then there exists a cubic blending surface $V(f)$ which is tangent to $V\left(g_{i}\right)$ along $V\left(g_{i}, h_{i}\right)(i=1,2)$. They expanded $g_{1}$ and $g_{2}$ with respect to $h_{1}, h_{2}$, and $h_{3}$, that is,

$$
\begin{aligned}
g_{i}= & \widehat{g}_{i, 21} h_{1}^{2}+\widehat{g}_{i, 22} h_{2}^{2}+\widehat{g}_{i, 23} h_{3}^{2}+\widehat{g}_{i, m 1} h_{2} h_{3}+\widehat{g}_{i, m 2} h_{1} h_{3}+\widehat{g}_{i, m 3} h_{1} h_{2} \\
& +\widehat{g}_{i, 11} h_{1}+\widehat{g}_{i, 12} h_{2}+\widehat{g}_{i, 13} h_{3}+\widehat{g}_{i, 0} \quad(i=1,2)
\end{aligned}
$$

and obtained the following lemma.

Lemma 2.3 (see [12]). If the coefficients of $g_{1}$ and $g_{2}$ defined by (2.1) satisfy

$$
\begin{gathered}
\frac{\widehat{g}_{1,23}}{\widehat{g}_{2,23}}=\frac{\widehat{g}_{1,13}}{\widehat{g}_{2,13}}=\frac{\widehat{g}_{1,0}}{\widehat{g}_{2,0}}=\kappa, \\
\frac{\widehat{g}_{1,11}-\kappa \widehat{g}_{2,11}}{\widehat{g}_{1,12}-\kappa \widehat{g}_{2,12}}=\frac{\widehat{g}_{1, m 2}-\kappa \widehat{g}_{2, m 2}}{\widehat{g}_{1, m 1}-\kappa \widehat{g}_{2, m 1}}=\mu,
\end{gathered}
$$

where $\kappa$ and $\mu$ are nonzero real numbers, then the cubic surface $V(f)$ defined by

$$
\begin{aligned}
f=\left(-\mu h_{1}+h_{2}\right) g_{1}+[ & \left(\widehat{g}_{1,21}-\kappa \widehat{g}_{2,21}\right)\left(\mu h_{1}-h_{2}\right)+\mu\left(\widehat{g}_{1, m 3}-\kappa \widehat{g}_{2, m 3}\right) h_{2} \\
+ & \left.\mu\left(\widehat{g}_{1, m 2}-\kappa \widehat{g}_{2, m 2}\right) h_{3}+\mu\left(\widehat{g}_{1,11}-\kappa \widehat{g}_{2,11}\right)\right] h_{1}^{2}
\end{aligned}
$$

meets $V\left(g_{i}\right)$ with $G^{1}$ continuity along $V\left(g_{i}, h_{i}\right)(i=1,2)$. surfaces:

Now, we consider successively two classes of these nonsingular cubic blending

(i) the nonsingular cubic surfaces $V(f)$ defined by (2.3), which are constructed under the above general assumptions, are called the general forms, 
(ii) if we add some extra restrictions to $V\left(g_{1}\right), V\left(g_{2}\right), V\left(h_{1}\right)$, and $V\left(h_{2}\right)$ :

(1) let $V\left(g_{1}\right)$ and $V\left(g_{2}\right)$ be quadratic surfaces defined by

$$
g_{i}=\left\{\begin{array}{l}
x_{j}^{2}+x_{k}^{2}-r_{i}^{2}, \\
\left(x_{i}-x_{i 0}\right)^{2}+x_{j}^{2}+x_{k}^{2}-r_{i}^{2}, \\
x_{j}^{2}+x_{k}^{2}-c_{i}^{2}\left(x_{i}-x_{i 0}\right)^{2}, \\
x_{j}^{2}+x_{k}^{2}-2 p_{i}\left(x_{i}-x_{i 0}\right), \\
x_{j}^{2}+x_{k}^{2}-c_{i}^{2}\left(x_{i}-x_{i 0}\right)^{2}-l_{i}^{2} \\
x_{j}^{2}+x_{k}^{2}-c_{i}^{2}\left(x_{i}-x_{i 0}\right)^{2}+l_{i}^{2}
\end{array} \quad(i=1,2)\right.
$$

where $\{j, k\}=\{1,2,3\} \backslash\{i\}$ and $r_{i}, x_{i 0}, c_{i}, p_{i}$, and $l_{i}$ are nonzero real numbers;

(2) let $V\left(h_{1}\right)$ and $V\left(h_{2}\right)$ be associated clipping planes defined by

$$
h_{i}=x_{i}+h_{i 0} \quad(i=1,2)
$$

where $h_{10}$ and $h_{20}$ are nonzero real numbers,

then the nonsingular cubic surfaces $V(f)$ defined by (2.3), which are constructed under this specific assumptions, are called the specific forms.

For these two classes of nonsingular cubic blending surfaces, our question is whether or not they have any good geometric property or any fast/exact parametrization algorithm.

\section{Nonsingular Cubic Blending Surfaces: The Specific Forms}

In this section, we mainly analyze some geometric information of the specific forms which is useful for the parametrization process and compute their uniform rational parametric representation.

\subsection{Geometric Information}

\subsubsection{Lines on the Surfaces}

According to the defining polynomial (2.3) and the results obtained in [13], we can easily find that $V\left(h_{1}, h_{2}\right)$ is just one line on the surfaces.

Let $h_{3}=x_{3}$. As showed in Section 2.1, we substitute the equation $h_{1}-\lambda h_{2}=0(\lambda$ is a parameter) of the pencil of planes into $f\left(h_{1}, h_{2}, h_{3}\right)=0$ and obtain

$$
Q\left(h_{2}, h_{3} ; \lambda\right)=A_{1, \lambda} h_{3}^{2}+A_{2, \lambda} h_{2}^{2}+A_{3, \lambda} h_{2}+A_{4, \lambda}=0,
$$

of the residual conic $C$ after cancelling the factor $h_{2}$. It is easy to demonstrate the following proposition by checking each pair of $V\left(g_{1}\right)$ and $V\left(g_{2}\right)$ defined by (2.4). 
Proposition 3.1. $A_{1, \lambda}, \ldots, A_{4, \lambda}$ in (3.1) satisfy that

(1) $A_{1, \lambda}, A_{2, \lambda}, A_{3, \lambda}, A_{4, \lambda}$ are polynomials of degree $1,3,2,1$ in $\lambda$, respectively,

(2) $A_{2, \lambda}=\left(\lambda^{2}+1\right) A_{1, \lambda}$,

(3) $A_{1, \lambda}$ and $A_{4, \lambda}$ differ by a nonzero constant.

We let

$$
\overline{\mathrm{D}}(\lambda):=\left|\begin{array}{cc}
A_{2, \lambda} & \frac{1}{2} A_{3, \lambda} \\
\frac{1}{2} A_{3, \lambda} & A_{4, \lambda}
\end{array}\right|=A_{2, \lambda} A_{4, \lambda}-\frac{1}{4} A_{3, \lambda}^{2}
$$

which is a polynomial of degree 4 in $\lambda$. Let $b_{i}$ denote the coefficient of $\lambda^{i}$ in $\overline{\mathrm{D}}(\lambda), i=0,1, \ldots, 4$, respectively. In other words, $\overline{\mathrm{D}}(\lambda)=b_{4} \lambda^{4}+b_{3} \lambda^{3}+b_{2} \lambda^{2}+b_{1} \lambda+b_{0}$.

The determinant of the Hessian of $Q\left(h_{2}, h_{3} ; \lambda\right)$ is

$$
\mathrm{D}(\lambda)=\left|\begin{array}{ccc}
A_{1, \lambda} & 0 & 0 \\
0 & A_{2, \lambda} & \frac{1}{2} A_{3, \lambda} \\
0 & \frac{1}{2} A_{3, \lambda} & A_{4, \lambda}
\end{array}\right|=A_{1, \lambda} \overline{\mathrm{D}}(\lambda)
$$

Since $\mathrm{D}(\lambda)$ factors into the product of $A_{1, \lambda}$ and $\overline{\mathrm{D}}(\lambda)$, now it is possible to solve the equation $\mathrm{D}(\lambda)=0$ analytically by some fixed formula. So, we can find all the exact lines on the surfaces by repeating the same process on the new lines that have been found.

\subsubsection{A Classification of the Surfaces}

Let $a+\mathrm{I} \cdot b$ be an arbitrary complex number, where $a, b \in \mathrm{R}$ and I denotes the imaginary unit. We formulate

$$
\sqrt{a+\mathrm{I} \cdot b}= \begin{cases}\frac{\sqrt{2}}{2}\left(\sqrt{\sqrt{a^{2}+b^{2}}+a}+\mathrm{I} \cdot \sqrt{\sqrt{a^{2}+b^{2}}-a}\right), & \text { if } b>0 \text { or } b=0 \text { and } a<0, \\ \frac{\sqrt{2}}{2}\left(\sqrt{\sqrt{a^{2}+b^{2}}+a}-\mathrm{I} \cdot \sqrt{\sqrt{a^{2}+b^{2}}-a}\right), & \text { if } b<0 \text { or } b=0 \text { and } a \geq 0 .\end{cases}
$$

The real and complex roots of the equation $D(\lambda)=0$ correspond to the real and complex tritangent planes of the surfaces, respectively. In the complex tritangent planes, $Q\left(h_{2}, h_{3} ; \lambda\right)$ factors into two complex lines, that is,

$$
Q\left(h_{2}, h_{3} ; \bar{\lambda}\right)=\left(\sqrt{A_{2, \bar{\lambda}}} h_{2}+\mathrm{I} \cdot \sqrt{A_{1, \bar{\lambda}}} h_{3}+\frac{A_{3, \bar{\lambda}}}{2 \sqrt{A_{2, \bar{\lambda}}}}\right)\left(\sqrt{A_{2, \bar{\lambda}}} h_{2}-\mathrm{I} \cdot \sqrt{A_{1, \bar{\lambda}}} h_{3}+\frac{A_{3, \bar{\lambda}}}{2 \sqrt{A_{2, \bar{\lambda}}}}\right),
$$


Table 2: A classification of nonsingular cubic surfaces (derived from [14]).

\begin{tabular}{lcccccc}
\hline Surface type & $F_{1}$ & $F_{3}$ & $F_{5}$ & $F_{2}$ & $F_{4}$ & $F_{3}$ \\
\hline Number of real roots of $\mathrm{D}(\lambda)=0$ & 5 & 5 & 5 & 3 & 3 & 1 \\
Number of real factorizations & 5 & 3 & 1 & 3 & 1 & 1 \\
\hline
\end{tabular}

where $\bar{\lambda}$ denotes a complex root of $\mathrm{D}(\lambda)=0$. However, in the real tritangent planes, some of the factorizations of $Q\left(h_{2}, h_{3} ; \lambda\right)$ are real, and some are complex.

Segre in [14] classifies nonsingular cubic surfaces using Table 2, according to the number of real roots of $\mathrm{D}(\lambda)=0$ and whether or not the factorization of $Q\left(h_{2}, h_{3} ; \lambda\right)$ in the corresponding tritangent plane is real or complex.

Using Table 2 and Proposition 3.1, we can arrive at the following theorem.

Theorem 3.2. The specific forms of nonsingular cubic blending surfaces must be $F_{3}, F_{4}$, or $F_{5}$ surfaces.

Proof. Let $\lambda_{1}$ be the root of $A_{1, \lambda}=0$. For all $\lambda \in R \backslash\left\{\lambda_{1}\right\}$, we have

$$
A_{1, \lambda} A_{2, \lambda}=\left(1+\lambda^{2}\right) A_{1, \lambda}^{2}>0
$$

The factorization of $Q\left(h_{2}, h_{3} ; \lambda\right)$ in a real tritangent plane is real if and only if there exists a $\lambda^{*} \in \mathrm{R}$ such that

$$
\mathrm{D}\left(\lambda^{*}\right)=0, \quad A_{1, \lambda^{*}} A_{2, \lambda^{*}} \leq 0 .
$$

Obviously, $\lambda_{1}$ is the only one which satisfies (3.7). Thus, the number of real factorizations in Table 2 is at most 1 , and this completes the proof.

In what follows, we will give a complete solution for the surface type problem of the surfaces. The main tool we will use is the complete discrimination system for any polynomial with real coefficients proposed by Yang et al. in [15].

Lemma 3.3 (see [15]). Given a polynomial $G(x)=a_{0} x^{m}+a_{1} x^{m-1}+\cdots+a_{m} \in R[x]$, if the number of sign changes in the revised sign list with respect to $G(x)$ is $v$, then the number of pairs of the distinct conjugate imaginary roots of $G(x)$ is $v$. Moreover, if the number of nonvanishing members in the revised sign list with respect to $G(x)$ is $\eta$, then the number of the distinct real roots is $\eta-2 v$.

Applying Lemma 3.3 to $\overline{\mathrm{D}}(\lambda)$ defined in (3.2) and according to Table 2 and Theorem 3.2, we can eventually arrive at the following proposition. 
Proposition 3.4. The discriminant sequence $\left[D_{1}, D_{2}, D_{3}, D_{4}\right]$ of $\bar{D}(\lambda)$ is of the form

$$
\begin{aligned}
D_{1}= & 1, \\
D_{2}= & -8 b_{2} b_{4}+3 b_{3}^{2}, \\
D_{3}= & \left(16 b_{0} b_{2}-18 b_{1}^{2}\right) b_{4}^{2}+\left(14 b_{1} b_{2} b_{3}-6 b_{0} b_{3}^{2}-4 b_{2}^{3}\right) b_{4}+b_{2}^{2} b_{3}^{2}-3 b_{1} b_{3}^{3}, \\
D_{4}= & 256 b_{0}^{3} b_{4}^{3}+\left(144 b_{0} b_{1}^{2} b_{2}-27 b_{1}^{4}-192 b_{0}^{2} b_{1} b_{3}-128 b_{0}^{2} b_{2}^{2}\right) b_{4}^{2} \\
& +\left(18 b_{1}^{3} b_{2} b_{3}-4 b_{1}^{2} b_{2}^{3}+16 b_{0} b_{2}^{4}-80 b_{0} b_{1} b_{2}^{2} b_{3}-6 b_{0} b_{1}^{2} b_{3}^{2}+144 b_{0}^{2} b_{2} b_{3}^{2}\right) b_{4} \\
& -4 b_{0} b_{2}^{3} b_{3}^{2}+18 b_{0} b_{1} b_{2} b_{3}^{3}-27 b_{0}^{2} b_{3}^{4}+b_{1}^{2} b_{2}^{2} b_{3}^{2}-4 b_{1}^{3} b_{3}^{3},
\end{aligned}
$$

where $b_{i}$ is the coefficient of $\lambda^{i}$ in $\bar{D}(\lambda), i=0,1, \ldots, 4$, respectively.

(1) If one of the following conditions holds:

$$
\begin{aligned}
D_{2}<0 \wedge D_{3}<0 \wedge D_{4}>0 ; & D_{2} \geq 0 \wedge D_{3} \leq 0 \wedge D_{4}>0 ; \\
D_{2}<0 \wedge D_{3} \geq 0 ; & D_{2}=0 \wedge D_{3}>0,
\end{aligned}
$$

then the specific forms of nonsingular cubic blending surfaces are $F_{3}$ surfaces.

(2) If one of the following conditions holds:

$$
\begin{gathered}
D_{2} \leq 0 \wedge D_{3}<0 \wedge D_{4} \leq 0 ; \quad D_{2}=0 \wedge D_{3}=0 \wedge D_{4}<0 \\
D_{2}>0 \wedge D_{3}<0 \wedge D_{4}=0 ; \quad D_{2}>0 \wedge D_{4}<0,
\end{gathered}
$$

then the specific forms of nonsingular cubic blending surfaces are $F_{4}$ surfaces.

(3) If one of the following conditions holds:

$$
D_{2}>0 \wedge D_{3}>0 \wedge D_{4} \geq 0 ; \quad D_{2} \geq 0 \wedge D_{3}=0 \wedge D_{4}=0,
$$

then the specific forms of nonsingular cubic blending surfaces are $F_{5}$ surfaces.

The above symbol " $\wedge$ " indicates logical conjunction, which means that $A \wedge B$ holds if and only if both $A$ and $B$ hold simultaneously.

\subsection{The Uniform Rational Parametric Representation}

Since our results of this subsection are based on the algorithm proposed by Berry and Patterson in [5], we first outline the strategy of their algorithm. 
Suppose that an $F_{i}(i=3,4)$ surface is given by $f\left(x_{1}, x_{2}, x_{3}\right)=0$.

(1) Find a line $l$ on the surface.

(2) Find a pair of complex conjugate tritangent planes through $l$, and denote them by $m$ and $m^{*}$.

(3) Compute the factorizations of the residual conic $\mathcal{C}$ (mentioned in Section 2.1) in $m$ and $m^{*}$, and denote them by $m_{1} \cdot m_{2}$ and $m_{1}^{*} \cdot m_{2}^{*}$, respectively.

(4) Construct a $3 \times 3$ complex matrix $\bar{U}$ of the form

$$
\left(\begin{array}{ccc}
m & 0 & m_{1} \\
0 & m^{*} & m_{1}^{*} \\
k m_{2} & k^{*} m_{2}^{*} & p
\end{array}\right)
$$

such that $k$ is a complex number; $p$ is a real plane; $\operatorname{det}(\bar{U})=f$.

(5) Find a real matrix $U$ which is equivalent to $\bar{U}$ and satisfies $\operatorname{det}(U)=f$.

(6) Compute the Hilbert-Burch matrix $H$ from the equation

$$
U\left(y_{1}, y_{2}, y_{3}\right)^{T}=H\left(x_{1}, x_{2}, x_{3}, 1\right)^{T}
$$

A rational parametrization of the surface $V(f)$ is

$$
x_{1}=-\frac{\operatorname{det}\left(H_{1}\right)}{\operatorname{det}\left(H_{4}\right)}, \quad x_{2}=\frac{\operatorname{det}\left(H_{2}\right)}{\operatorname{det}\left(H_{4}\right)}, \quad x_{3}=-\frac{\operatorname{det}\left(H_{3}\right)}{\operatorname{det}\left(H_{4}\right)},
$$

where $H_{i}$ are the $3 \times 3$ submatrices of $H$ by cancelling the $i$ th column.

Now, using the notations and conclusions in Section 3.1, we apply the algorithm to the specific forms of type $F_{3}$ and $F_{4}$.

According to Theorem 3.2, we can only choose a pair of complex conjugate roots, denoted by $\alpha$ and $\alpha^{*}$, of the equation $\mathrm{D}(\lambda)=0$. In the two corresponding complex tritangent planes, the factorizations of $Q\left(h_{2}, h_{3} ; \alpha\right)$ and $Q\left(h_{2}, h_{3} ; \alpha^{*}\right)$ must be the form (3.5). Thus, we can construct the uniform $3 \times 3$ complex matrix $\bar{U}$ of the form

$$
\left(\begin{array}{ccc}
h_{1}-\alpha h_{2} & 0 & \sqrt{A_{2, \alpha}} h_{2}+\mathrm{I} \cdot \sqrt{A_{1, \alpha}} h_{3}+\frac{A_{3, \alpha}}{2 \sqrt{A_{2, \alpha}}} \\
0 & h_{1}-\alpha^{*} h_{2} & \sqrt{A_{2, \alpha^{*}}} h_{2}-\mathrm{I} \cdot \sqrt{A_{1, \alpha^{*}}} h_{3}+\frac{A_{3, \alpha^{*}}}{2 \sqrt{A_{2, \alpha^{*}}}}
\end{array}\right),
$$


where

$$
\begin{aligned}
& \bar{u}_{31}=k\left(\sqrt{A_{2, \alpha}} h_{2}-\mathrm{I} \cdot \sqrt{A_{1, \alpha}} h_{3}+\frac{A_{3, \alpha}}{2 \sqrt{A_{2, \alpha}}}\right), \\
& \bar{u}_{32}=k^{*}\left(\sqrt{A_{2, \alpha^{*}}} h_{2}+\mathrm{I} \cdot \sqrt{A_{1, \alpha^{*}}} h_{3}+\frac{A_{3, \alpha^{*}}}{2 \sqrt{A_{2, \alpha^{*}}}}\right) .
\end{aligned}
$$

Proposition 3.5. $p_{0}, \ldots, p_{3}$, and $k$ in $\bar{U}$ are given by

$$
\begin{gathered}
p_{0}=-\mu \kappa \widehat{g}_{2,11}, \quad p_{1}=-\mu \kappa \widehat{g}_{2,21}, \quad p_{3}=-\mu \kappa \widehat{g}_{2, m 2}, \\
p_{2}=-\mu \kappa\left(\alpha+\alpha^{*}\right) \widehat{g}_{2,21}+\kappa\left(\widehat{g}_{2,21}-\mu \widehat{g}_{2, m 3}\right), \\
k=\frac{1}{A_{2, \alpha} A_{3, \alpha^{*}}-A_{2, \alpha^{*}} A_{3, \alpha}}\left[\left(\alpha+\alpha^{*}\right)\left(p_{0} A_{2, \alpha^{*}}-p_{2} A_{3, \alpha^{*}}\right)+\alpha \alpha^{*} p_{1} A_{3, \alpha^{*}}\right. \\
\left.+\left(\widehat{g}_{1,11}-\mu \widehat{g}_{1,12}\right) A_{2, \alpha^{*}}-\left(\widehat{g}_{1, m 3}-\mu \widehat{g}_{1,22}\right) A_{3, \alpha^{*}}\right] .
\end{gathered}
$$

Similarly, the uniform Hilbert-Burch matrix $H$ can be constructed. We let $\operatorname{Re}(z)$ and $\operatorname{Im}(z)$ denote the real part and the imaginary part of the complex number $z$, respectively.

Proposition 3.6. The uniform Hilbert-Burch matrix $H$ is of the form

$$
\left(\begin{array}{cccc}
2 y_{1} & -2 \operatorname{Re}(\alpha) y_{1}+\operatorname{Im}(\alpha) y_{2}+2 \operatorname{Re}\left(\sqrt{A_{2, \alpha}}\right) y_{3} & -2 \operatorname{Im}\left(\sqrt{A_{1, \alpha}}\right) y_{3} & \operatorname{Re}\left(\frac{A_{3, \alpha}}{\sqrt{A_{2, \alpha}}}\right) y_{3} \\
\frac{1}{2} y_{2} & -\operatorname{Im}(\alpha) y_{1}-\frac{1}{2} \operatorname{Re}(\alpha) y_{2}+\operatorname{Im}\left(\sqrt{A_{2, \alpha}}\right) y_{3} & \operatorname{Re}\left(\sqrt{A_{1, \alpha}}\right) y_{3} & \operatorname{Im}\left(\frac{A_{3, \alpha}}{\sqrt{A_{2, \alpha}}}\right) y_{3} \\
p_{1} y_{3} & 2 \operatorname{Re}\left(k \sqrt{A_{2, \alpha}}\right) y_{1}-\operatorname{Im}\left(k \sqrt{A_{2, \alpha}}\right) y_{2}+p_{2} y_{3} & e_{33} & e_{34}
\end{array}\right),
$$

where

$$
\begin{aligned}
& e_{33}=2 \operatorname{Im}\left(k \sqrt{A_{1, \alpha}}\right) y_{1}+\operatorname{Re}\left(k \sqrt{A_{1, \alpha}}\right) y_{2}+p_{3} y_{3}, \\
& e_{34}=\operatorname{Re}\left(k \frac{A_{3, \alpha}}{\sqrt{A_{2, \alpha}}}\right) y_{1}-\frac{1}{2} \operatorname{Im}\left(k \frac{A_{3, \alpha}}{\sqrt{A_{2, \alpha}}}\right) y_{2}+p_{0} y_{3} .
\end{aligned}
$$
$F_{3}$ and $F_{4}$ is

Finally, the uniform rational parametric representation for the specific forms of type

$$
x_{1}=-\frac{\operatorname{det}\left(H_{1}\right)+h_{10} \operatorname{det}\left(H_{4}\right)}{\operatorname{det}\left(H_{4}\right)}, \quad x_{2}=\frac{\operatorname{det}\left(H_{2}\right)-h_{20} \operatorname{det}\left(H_{4}\right)}{\operatorname{det}\left(H_{4}\right)}, \quad x_{3}=-\frac{\operatorname{det}\left(H_{3}\right)}{\operatorname{det}\left(H_{4}\right)},
$$

where $H_{i}$ are the $3 \times 3$ submatrices of (3.18) by cancelling the $i$ th column. 


\section{Nonsingular Cubic Blending Surfaces: The General Forms}

In this section, we will see that for the general forms, their parametrizations can also be computed using the symbolic computation. However, their parametrizations are too complicated to be written down in a uniform form.

Similarly as the analysis in Section 3, we can easily come to the following results. According to the defining polynomial (2.3),

(i) $V\left(h_{1}, h_{2}\right)$ is just one line on the surfaces,

(ii) the equation of the residual conic $\mathcal{C}$ is

$$
Q\left(h_{2}, h_{3} ; \lambda\right)=a_{11} h_{3}^{2}+a_{12} h_{3} h_{2}+a_{22} h_{2}^{2}+a_{13} h_{3}+a_{23} h_{2}+a_{33}=0,
$$

where

$$
\begin{aligned}
& a_{11}=\widehat{g}_{1,23}(1-\mu \lambda), \\
& a_{12}=\widehat{g}_{1, m 1}(1-\mu \lambda)+\left(\widehat{g}_{1, m 2}-\mu \kappa \widehat{g}_{2, m 2} \lambda\right) \lambda, \\
& a_{22}=(1-\mu \lambda)\left(\kappa \widehat{g}_{2,21} \lambda^{2}+\widehat{g}_{1, m 3} \lambda+\widehat{g}_{1,22}\right)+\mu\left(\widehat{g}_{1, m 3}-\kappa \widehat{g}_{2, m 3}\right) \lambda^{2}, \\
& a_{13}=\widehat{g}_{1,13}(1-\mu \lambda), \\
& a_{23}=\widehat{g}_{1,12}(1-\mu \lambda)+\left(\widehat{g}_{1,11}-\mu \kappa \widehat{g}_{2,11} \lambda\right) \lambda, \\
& a_{33}=(1-\mu \lambda) \widehat{g}_{1,0},
\end{aligned}
$$

(iii) the determinant of the Hessian of $Q\left(h_{2}, h_{3} ; \lambda\right)$ is

$$
\mathrm{D}(\lambda)=\left|\begin{array}{lll}
a_{11} & \frac{1}{2} a_{12} & \frac{1}{2} a_{13} \\
\frac{1}{2} a_{12} & a_{22} & \frac{1}{2} a_{23} \\
\frac{1}{2} a_{13} & \frac{1}{2} a_{23} & a_{33}
\end{array}\right|=(1-\mu \lambda)\left(T_{4} \lambda^{4}+\cdots\right),
$$

where the second factor in the above product is some certain polynomial of degree 4 in $\lambda$. Thus we can solve the equation $D(\lambda)=0$ analytically,

(iv) at each root of $\mathrm{D}(\lambda)=0$, we discuss the sign of the minor

$$
\left|\begin{array}{ll}
a_{11} & \frac{1}{2} a_{12} \\
\frac{1}{2} a_{12} & a_{22}
\end{array}\right|,
$$

of (4.3). Then, we can determine the surface type and other geometric information using Table 2 and Table 1. 
Because of the existence of the mixed term $h_{3} h_{2}$ in (4.1), the uniform representation of the factorization of (4.1) is very complicated, and so, we omit it. Finally, using the algorithm in [5], the rational parametrization of the general forms can be achieved without approximate calculation.

\section{Example}

Example 5.1. We are given a nonsingular cubic blending surface $V(f)$ defined by the implicit equation

$$
\begin{aligned}
f\left(x_{1}, x_{2}, x_{3}\right)= & x_{1}^{3}+x_{1}^{2} x_{2}+x_{1} x_{2}^{2}+x_{1} x_{3}^{2}+x_{2}^{3}+x_{2} x_{3}^{2}-6 x_{1}^{2}-12 x_{1} x_{2}-6 x_{2}^{2} \\
& -6 x_{3}^{2}+21 x_{1}+21 x_{2}-18=0,
\end{aligned}
$$

and we also know that $V(f)$ meets the circular cone $V\left(g_{i}\right)$ with $G^{1}$ continuity along $V\left(g_{i}, h_{i}\right)$, $i=1,2$, where

$$
\begin{array}{ll}
g_{1}=x_{2}^{2}+x_{3}^{2}-\frac{3}{2}\left(x_{1}-1\right)^{2}, & h_{1}=x_{1}-3, \\
g_{2}=x_{1}^{2}+x_{3}^{2}-\frac{3}{2}\left(x_{2}-1\right)^{2}, & h_{2}=x_{2}-3 .
\end{array}
$$

First, we let $h_{3}=x_{3}$, and rewrite $f\left(x_{1}, x_{2}, x_{3}\right)=0$ in the following form:

$$
f\left(h_{1}, h_{2}, h_{3}\right)=\left(h_{1}+h_{2}\right)\left[\left(h_{2}+3\right)^{2}+h_{3}^{2}-\frac{3}{2}\left(h_{1}+2\right)^{2}\right]+\left(\frac{5}{2} h_{1}+\frac{5}{2} h_{2}+12\right) h_{1}^{2}=0
$$

\section{Geometric Information}

$V\left(h_{1}, h_{2}\right)$ is a line on $V(f)$. The equation of the residual conic $\mathcal{C}$ is

$$
Q\left(h_{2}, h_{3} ; \lambda\right)=(\lambda+1) h_{3}^{2}+(\lambda+1)\left(\lambda^{2}+1\right) h_{2}^{2}+6\left(\lambda^{2}+1\right) h_{2}+3(\lambda+1)=0
$$

The determinant of the Hessian of $Q\left(h_{2}, h_{3} ; \lambda\right)$ is

$$
\mathrm{D}(\lambda)=(\lambda+1) \overline{\mathrm{D}}(\lambda)=-6(\lambda+1)\left(\lambda^{2}+1\right)\left(\lambda^{2}-\lambda+1\right) .
$$

The discriminant sequence $\left[D_{1}, D_{2}, D_{3}, D_{4}\right]$ of $\overline{\mathrm{D}}(\lambda)$ satisfies that

$$
D_{1}=1, \quad D_{2}=-468<0, \quad D_{3}=6480>0, \quad D_{4}=559872>0 .
$$

According to Proposition 3.4, we know that $V(f)$ is an $F_{3}$ surface and other information of $V(f)$ from Table 1. 
Journal of Applied Mathematics

\section{Rational Parametrization}

We choose a pair of complex conjugate roots of $\mathrm{D}(\lambda)=0$, I and -I. As mentioned in Section 3.2, we construct the complex matrix $\bar{U}$ as

$$
\left(\begin{array}{ccc}
h_{1}-\mathrm{I} \cdot h_{2} & 0 & (1+\mathrm{I})\left(h_{3}+\sqrt{3} \cdot \mathrm{I}\right) \\
0 & h_{1}+\mathrm{I} \cdot h_{2} & (1-\mathrm{I})\left(h_{3}-\sqrt{3} \cdot \mathrm{I}\right) \\
\frac{1}{2} \cdot \mathrm{I} \cdot\left(h_{3}-\sqrt{3} \cdot \mathrm{I}\right) & -\frac{1}{2} \cdot \mathrm{I} \cdot\left(h_{3}+\sqrt{3} \cdot \mathrm{I}\right) & h_{1}+h_{2}+6
\end{array}\right)
$$

and the Hilbert-Burch matrix $H$ as

$$
\left(\begin{array}{cccc}
2 y_{1} & y_{2} & 2 y_{3} & -2 \sqrt{3} y_{3} \\
\frac{1}{2} y_{2} & -y_{1} & y_{3} & \sqrt{3} y_{3} \\
y_{3} & y_{3} & -\frac{1}{2} y_{2} & \sqrt{3} y_{1}+6 y_{3}
\end{array}\right)
$$

Finally, the rational parametrization of $V(f)$ is

$$
\begin{aligned}
& x_{1}=\frac{3 y_{1}^{2} y_{2}-2 \sqrt{3} y_{1}^{2} y_{3}-12 y_{1} y_{3}^{2}+(3 / 4) y_{2}^{3}-(\sqrt{3} / 2) y_{2}^{2} y_{3}-4 \sqrt{3} y_{3}^{3}}{y_{1}^{2} y_{2}+(1 / 4) y_{2}^{3}+2 y_{2} y_{3}^{2}}, \\
& x_{2}=\frac{3 y_{1}^{2} y_{2}+2 \sqrt{3} y_{1}^{2} y_{3}+12 y_{1} y_{3}^{2}+(3 / 4) y_{2}^{3}+(\sqrt{3} / 2) y_{2}^{2} y_{3}+4 \sqrt{3} y_{3}^{3}}{y_{1}^{2} y_{2}+(1 / 4) y_{2}^{3}+2 y_{2} y_{3}^{2}}, \\
& x_{3}=\frac{2 \sqrt{3} y_{1}^{3}+12 y_{1}^{2} y_{3}+(\sqrt{3} / 2) y_{1} y_{2}^{2}+4 \sqrt{3} y_{1} y_{3}^{2}+3 y_{2}^{2} y_{3}}{y_{1}^{2} y_{2}+(1 / 4) y_{2}^{3}+2 y_{2} y_{3}^{2}} .
\end{aligned}
$$

Example 5.2. We are given a nonsingular cubic blending surface $V(f)$ defined by the implicit equation

$$
\begin{aligned}
f\left(x_{1}, x_{2}, x_{3}\right)= & x_{1}^{3}+4 x_{1}^{2} x_{2}+4 x_{1}^{2} x_{3}+6 x_{1} x_{2}^{2}+12 x_{1} x_{2} x_{3}+7 x_{1} x_{3}^{2}+4 x_{2}^{3}+12 x_{2}^{2} x_{3} \\
& +14 x_{2} x_{3}^{2}+6 x_{3}^{3}-2 x_{1}^{2}-8 x_{1} x_{2}-6 x_{1} x_{3}-8 x_{2}^{2}-12 x_{2} x_{3}-8 x_{3}^{2} \\
& -x_{1}-2 x_{2}-10 x_{3}+4=0
\end{aligned}
$$

and we also know that $V(f)$ meets the quadratic $V\left(g_{i}\right)$ with $G^{1}$ continuity along $V\left(g_{i}, h_{i}\right)$, $i=1,2$, where

$$
\begin{gathered}
g_{1}=x_{1}^{2}+2 x_{1} x_{2}+2 x_{1} x_{3}+2 x_{2}^{2}+4 x_{2} x_{3}+3 x_{3}^{2}-6 x_{1}-4 x_{2}-2 x_{3}+7, \\
g_{2}=x_{1}^{2}+2 x_{1} x_{2}+2 x_{1} x_{3}+2 x_{2}^{2}+4 x_{2} x_{3}+3 x_{3}^{2}+2 x_{1}-4 x_{2}-2 x_{3}+7, \\
h_{1}=x_{1}+x_{2}+x_{3}-2, \quad h_{2}=x_{2}+x_{3}-2 .
\end{gathered}
$$


First, we let $h_{3}=x_{3}$ and rewrite $f\left(x_{1}, x_{2}, x_{3}\right)=0$ in the following form:

$$
f\left(h_{1}, h_{2}, h_{3}\right)=\left(h_{1}+h_{2}\right)\left[\left(h_{2}+3\right)^{2}+\left(h_{3}+1\right)^{2}+\left(h_{1}-1\right)^{2}-4\right]+8 h_{1}^{2}=0 .
$$

\section{Geometric Information}

$V\left(h_{1}, h_{2}\right)$ is a line on $V(f)$. The equation of the residual conic $\mathcal{C}$ is

$$
\begin{aligned}
Q\left(h_{2}, h_{3} ; \lambda\right)= & (\lambda+1) h_{3}^{2}+(\lambda+1)\left(\lambda^{2}+1\right) h_{2}^{2}+2(\lambda+1) h_{3} \\
& +\left(6 \lambda^{2}+4 \lambda+6\right) h_{2}+7(\lambda+1)=0 .
\end{aligned}
$$

The determinant of the Hessian of $Q\left(h_{2}, h_{3} ; \lambda\right)$ is

$$
\begin{aligned}
D(\lambda) & =\left|\begin{array}{ccc}
\lambda+1 & 0 & \lambda+1 \\
0 & (\lambda+1)\left(\lambda^{2}+1\right) & 3 \lambda^{2}+2 \lambda+3 \\
\lambda+1 & 3 \lambda^{2}+2 \lambda+3 & 7(\lambda+1)
\end{array}\right| \\
& =-(\lambda+1)\left(3 \lambda^{4}+10 \lambda^{2}+3\right) .
\end{aligned}
$$

The discriminant sequence $\left[D_{1}, D_{2}, D_{3}, D_{4}\right]$ of $-\left(3 \lambda^{4}+10 \lambda^{2}+3\right)$ satisfies that

$$
D_{1}=1, \quad D_{2}=-240<0, \quad D_{3}=-7680<0, \quad D_{4}=589824>0 .
$$

According to Lemma 3.3 and the coefficients of $h_{3}^{2}$ and $h_{2}^{2}$ in $Q\left(h_{2}, h_{3} ; \lambda\right)$, we know that $V(f)$ is an $F_{3}$ surface and other information of $V(f)$ from Table 1.

\section{Rational Parametrization}

We choose a pair of complex conjugate roots of $\mathrm{D}(\lambda)=0, \sqrt{3} \cdot \mathrm{I}$ and $-\sqrt{3} \cdot \mathrm{I}$. We can construct the complex matrix $\bar{U}$ as

$$
\left(\begin{array}{ccc}
h_{1}-\sqrt{3} \cdot \mathrm{I} \cdot h_{2} & 0 & (1+\sqrt{3} \cdot \mathrm{I})\left(h_{3}+\sqrt{2} h_{2}+1-\sqrt{6} \cdot \mathrm{I}\right) \\
0 & h_{1}+\sqrt{3} \cdot \mathrm{I} \cdot h_{2} & (1-\sqrt{3} \cdot \mathrm{I})\left(h_{3}+\sqrt{2} h_{2}+1+\sqrt{6} \cdot \mathrm{I}\right) \\
\bar{u}_{31} & \bar{u}_{32} & h_{1}+h_{2}+6
\end{array}\right)
$$

where

$$
\begin{aligned}
& \bar{u}_{31}=\frac{\sqrt{3}}{6} \cdot \mathrm{I} \cdot\left(h_{3}-\sqrt{2} h_{2}+1+\sqrt{6} \cdot \mathrm{I}\right), \\
& \bar{u}_{32}=-\frac{\sqrt{3}}{6} \cdot \mathrm{I} \cdot\left(h_{3}-\sqrt{2} h_{2}+1-\sqrt{6} \cdot \mathrm{I}\right),
\end{aligned}
$$


and the Hilbert-Burch matrix $H$ as

$$
\left(\begin{array}{cccc}
2 y_{1} & \sqrt{3} y_{2}+2 \sqrt{2} y_{3} & 2 y_{3} & (2+6 \sqrt{2}) y_{3} \\
\frac{1}{2} y_{2} & -\sqrt{3} y_{1}+\sqrt{6} y_{3} & \sqrt{3} y_{3} & (1-\sqrt{6}) y_{3} \\
y_{3} & \frac{\sqrt{6}}{6} y_{2}+y_{3} & -\frac{\sqrt{3}}{6} y_{2} & -\sqrt{2} y_{1}-\frac{\sqrt{3}}{6} y_{2}+6 y_{3}
\end{array}\right) .
$$

Finally, the rational parametrization of $V(f)$ is

$$
\left(x_{1}: x_{2}: x_{3}\right)=\left(\frac{Y_{1}}{Y_{4}}: \frac{Y_{2}}{Y_{4}}: \frac{Y_{3}}{Y_{4}}\right)
$$

where

$$
\begin{aligned}
Y_{1}= & 4 \sqrt{6} y_{1}^{2} y_{3}-24 \sqrt{3} y_{1} y_{3}^{2}+\sqrt{6} y_{2}^{2} y_{3}+4 y_{2} y_{3}^{2}+16 \sqrt{6} y_{3}^{3}, \\
Y_{2}= & 2 \sqrt{6} y_{1}^{3}+3 y_{1}^{2} y_{2}-(2 \sqrt{6}+16 \sqrt{3}) y_{1}^{2} y_{3}+\frac{\sqrt{6}}{2} y_{1} y_{2}^{2}+(4-6 \sqrt{2}) y_{1} y_{2} y_{3} \\
& +(12 \sqrt{3}+20 \sqrt{6}) y_{1} y_{3}^{2}+\frac{3}{4} y_{2}^{3}+\left(\frac{\sqrt{6}}{2}-2 \sqrt{3}\right) y_{2}^{2} y_{3}+(6-6 \sqrt{2}) y_{2} y_{3}^{2} \\
& -(8 \sqrt{6}+16 \sqrt{3}) y_{3}^{3} \\
Y_{3}= & -2 \sqrt{6} y_{1}^{3}-y_{1}^{2} y_{2}+16 \sqrt{3} y_{1}^{2} y_{3}-\frac{\sqrt{6}}{2} y_{1} y_{2}^{2}+(2 \sqrt{2}-4) y_{1} y_{2} y_{3}-20 \sqrt{6} y_{1} y_{3}^{2} \\
& -\frac{1}{4} y_{2}^{3}+\left(2 \sqrt{3}-\frac{\sqrt{6}}{3}\right) y_{2}^{2} y_{3}+(6 \sqrt{2}-4) y_{2} y_{3}^{2}+16 \sqrt{3} y_{3}^{3}, \\
Y_{4}= & y_{1}^{2} y_{2}-2 \sqrt{2} y_{1} y_{2} y_{3}+\frac{1}{4} y_{2}^{3}+\frac{\sqrt{6}}{3} y_{2}^{2} y_{3}+4 y_{2} y_{3}^{2} .
\end{aligned}
$$

\section{Conclusions}

This paper is concerned with nonsingular cubic blending surfaces. We mainly discuss geometric information and rational parametrization of them. Since their underlying geometric properties, the rational parametrization can be implemented using the symbolic computation, while for general nonsingular cubic surfaces, it has to resort to floating point numbers. In the future, we will focus on the analysis of singular cubic blending surfaces and nonsingular cubic blending surfaces of type $F_{5}$.

\section{Acknowledgments}

The authors express their deep gratitude to the referee for useful suggestions and comments which have improved the paper. This work is partly supported by National Science Foundation of China (Grant no. 60973155). 


\section{References}

[1] A. Henderson, The Twenty-Seven Lines Upon the Cubic Surface, Hafner Publishing, New York, NY, USA, 1911.

[2] T. Sederberg and J. Snively, "Parametrization of cubic algebraic surfaces," in The Mathematics of Surfaces II, R. Martin, Ed., vol. 11, pp. 299-319, Clarendon Press, Oxford, UK, 1987.

[3] C. Bajaj, R. Holt, and A. Netravali, "Rational parameterizations of nonsingular real cubic-surfaces," ACM Transactions on Graphics, vol. 17, no. 1, pp. 1-31, 1998.

[4] I. Polo-Blanco and J. Top, "A remark on parameterizing nonsingular cubic surfaces," Computer Aided Geometric Design, vol. 26, no. 8, pp. 842-849, 2009.

[5] T. Berry and R. Patterson, "Implicitization and parametrization of nonsingular cubic surfaces," Computer Aided Geometric Design, vol. 18, no. 8, pp. 723-738, 2001.

[6] F. Chen, L. Shen, and J. Deng, "Implicitization and parametrization of quadratic and cubic surfaces by $\mu$-bases," Computing, vol. 79, no. 2-4, pp. 131-142, 2007.

[7] T. Wu, W. Gao, and G. Feng, "Blending of implicit algebraic surfaces," in Proceedings of the ASCM, pp. 125-131, Beijing, China, August 1995.

[8] T. Wu and Y. Zhou, "On blending of several quadratic algebraic surfaces," Computer Aided Geometric Design, vol. 17, no. 8, pp. 759-766, 2000.

[9] N. Lei, Blending of two quadratic surfaces and Wu Wen-tsün's formulae, Ph.D. thesis, Jilin University, Jilin, China, 2002.

[10] T. Wu and H. Cheng, "Basic lines, axes and geometric modeling on implicit algebraic surfaces," Journal of Computational and Applied Mathematics, vol. 195, no. 1-2, pp. 212-219, 2006.

[11] T. Sederberg, "Techniques for cubic algebraic surfaces II," IEEE Computer Graphics and Applications, vol. 10, no. 5, pp. 12-21, 1990.

[12] T. Wu, N. Lei, and J. Cheng, “Wu Wen-tsün's formulae for the blending of pipe surfaces," Northeastern Mathematical Journal, vol. 17, no. 4, pp. 383-386, 2001.

[13] C. Li, A software system on blending of pipe surfaces, M.S. thesis, Jilin University, Jilin, China, 2000.

[14] B. Segre, The Non-Singular Cubic Surfaces, Oxford University Press, Oxford, UK, 1942.

[15] L. Yang, J. Zeng, and X. Hou, "A complete discrimination system for polynomials," Science in China, vol. E39, no. 6, pp. 628-646, 1996. 


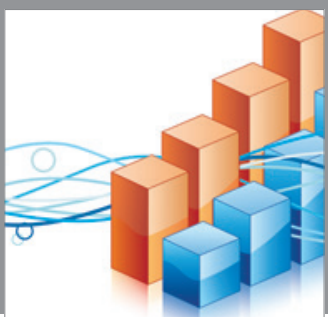

Advances in

Operations Research

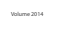

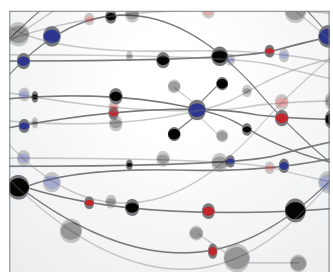

\section{The Scientific} World Journal
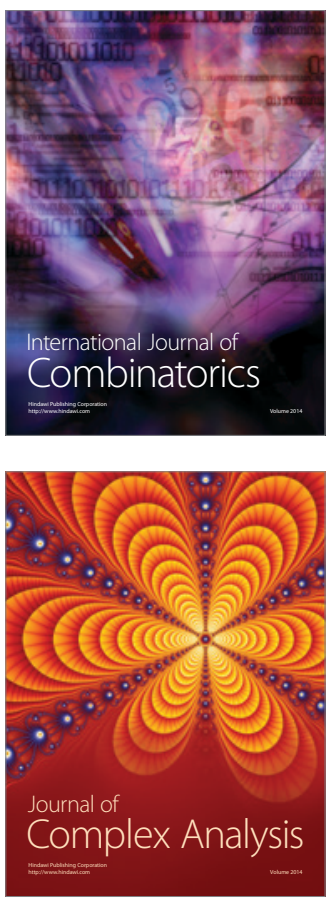

International Journal of

Mathematics and

Mathematical

Sciences
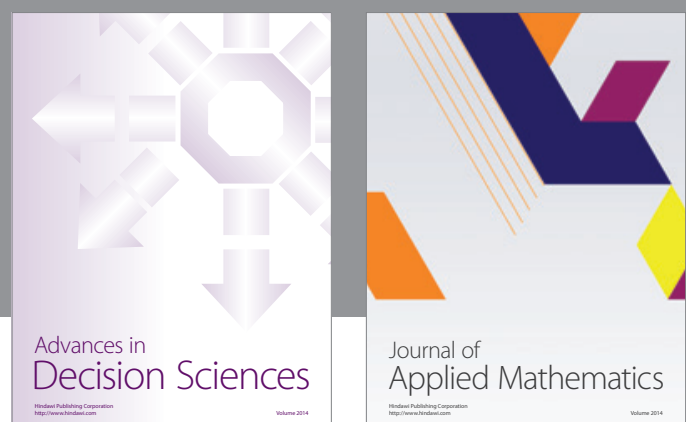

Journal of

Applied Mathematics
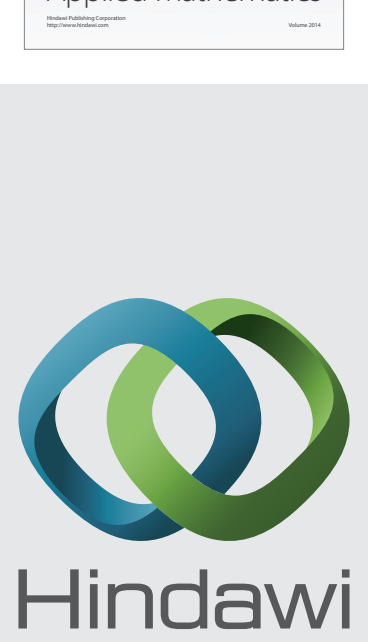

Submit your manuscripts at http://www.hindawi.com
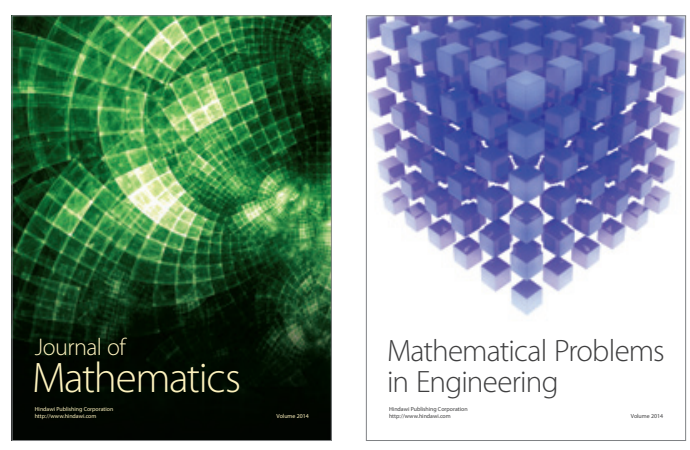

Mathematical Problems in Engineering
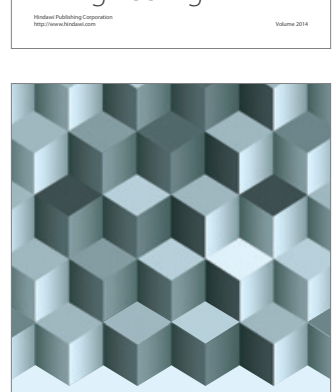

Journal of

Function Spaces
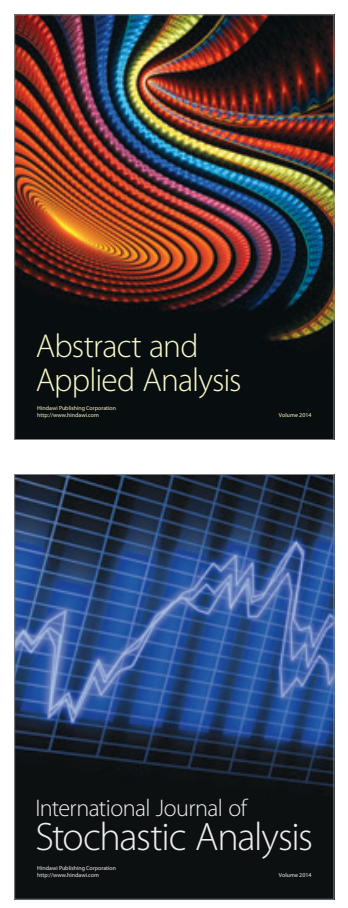

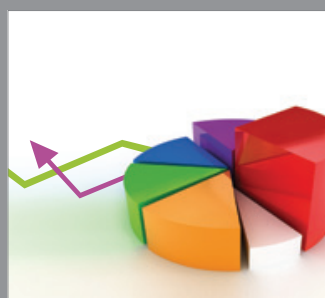

ournal of

Probability and Statistics

Promensencen
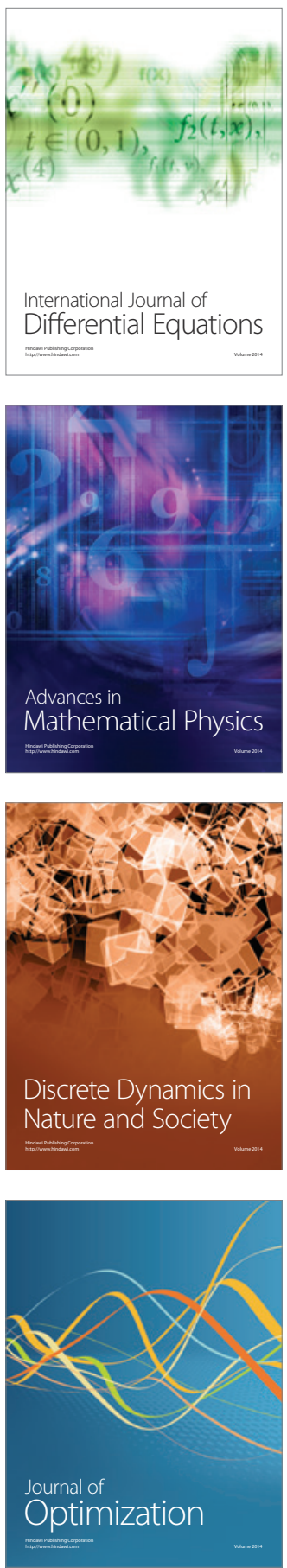